\title{
Magical mantle tour
}

\section{Geophysicists are racing to understand a recently discovered phenomenon deep in the Earth. David Cyranoski joins them.}

t's a place where everything goes weird. Some 2,700 kilometres inside the Earth, just above the boundary between the planet's solid mantle and its liquid outer core, is a layer where seismic waves act strangely. Instead of carrying on through the Earth, some waves speed up when they enter the zone and, once inside, can vary in speed depending on their direction of travel. In some pockets, they can slow by as much as $30 \%$.

Such seismic waves are scientists' primary means of probing Earth's depths; waves bouncing through the planet's interior have revealed how Earth is layered into crust, mantle, outer core and inner core. But what could account for the seismic oddness of this region, known as the $\mathrm{D}^{\prime \prime}$ (or D-double-prime) layer?

Until recently, nobody even knew what questions to ask. "A couple of years ago, deepEarth seismology seemed stagnant, ${ }^{n}$ says Christine Thomas, a seismologist at the University of Liverpool. But in 2004, a group led by Kei Hirose at the Tokyo Institute of Technology reported that they had reproduced the high pressures and temperatures of the lowermost mantle in the laboratory (see "A collaboration on the rocks"). And they found that the predominant mineral there a magnesium silicate, $\mathrm{MgSiO}_{3}-$ can take on a previously unknown structure ${ }^{1}$.

The structure, known as the 'post-perovskite phase' of $\mathrm{MgSiO}_{3}$, has the same mineralogical composition as the more common perovskite form, but its atoms line up differently. ${ }^{\alpha}$ The denser post-perovskite is like a stack of two-dimensional sheets, compared with the three-dimensional bonding structure of perovskite," says Hirose (see graphic). And that shift in atomic arrangement, known as a phase transition, could help explain the seismic oddities of Earth's lower mantle.

\section{Is that it?}

Phase transitions are common in geology, and to some Hirose's explanation seems disappointingly mundane. ${ }^{\alpha}$ What we were searching for all those years was nothing more than a phase transition," says John Brodholt, a mineral physicist at University College London. Nevertheless, post-perovskite has energized seismology, geodynamics and mineral physics.

In a flurry of publications, researchers have debated the structure's properties, what other seismological mysteries it might explain, and its implications for heat flow within the mantle. Planetary experts are re-examining some quirks of Earth's rotation in light of the postperovskite discovery, and are even thinking about how it could explain features of other

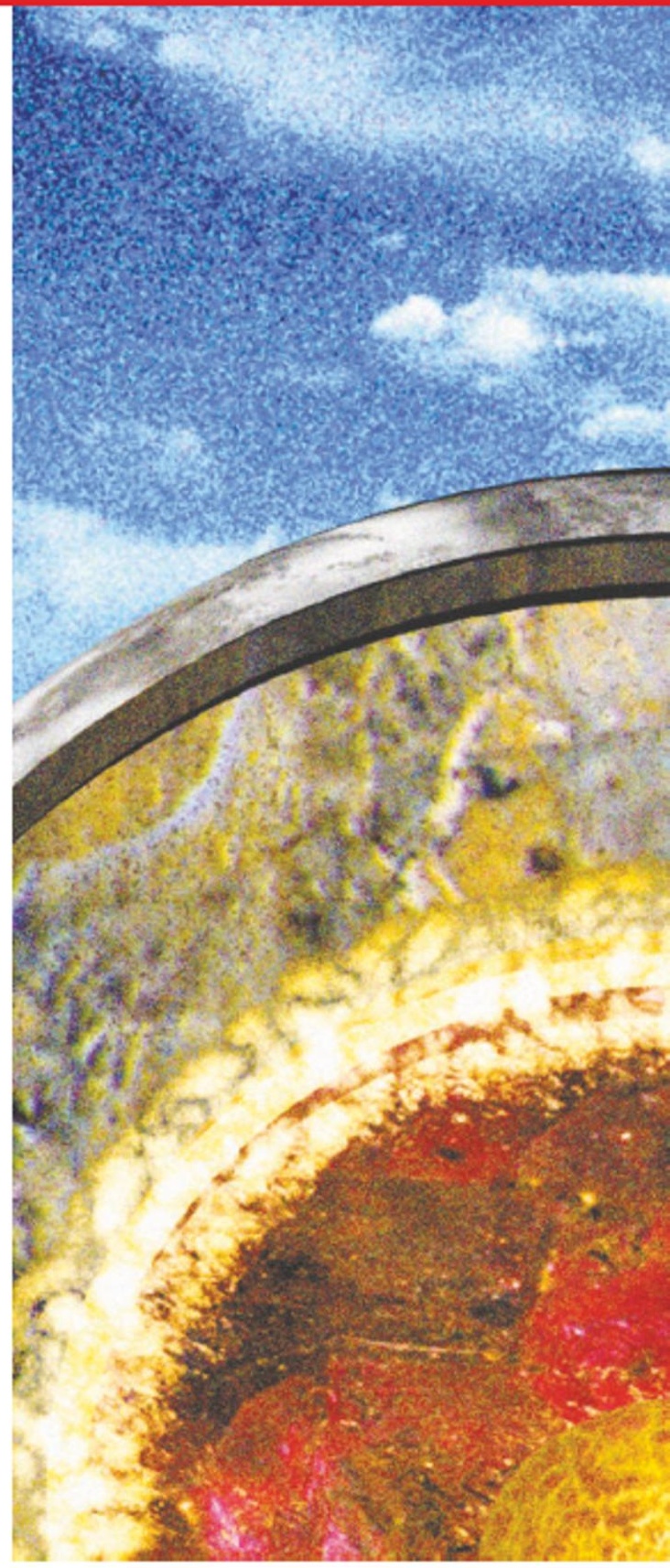

\section{A COLLABORATION ON THE ROCKS}

Post-perovskite $\mathrm{MgSiO}_{3}$ was a long-anticipated discovery. How did it finally happen? That depends on whom you ask.

KeiHirose and Shigeaki Ono used to be collaborators. Now, aside from polite greetings in the hallways, they don't talk.

They work one floor apartat Tokyo Institute of Technology, where Hirose is a professor and Ono, a researcher at the Japan Marine Science and Technology Center, collaborateswith another scientist. Both Hirose and Ono do similar work: squeezing materials, heated by a laser, betweentwo diamonds in a device called a diamond anvil. Both observe how the materials' properties change under extreme pressure, in conditions thatmimic Earth's interior.

Both Hirose and Ono made important contributions to the discovery of the post-perovskite phase of the mineral $\mathrm{MgSiO}_{3}$. Yet when Hirose organized a conference on the topic in Tokyo last October, Ono wasn't invited to speak.

Hirose is outgoing, talkative and animated; he commanded the workshop with confident English that he had learnt during a

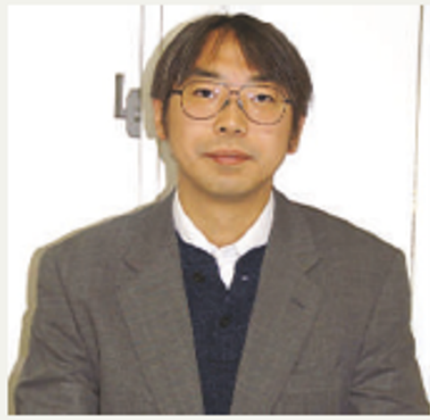

Kei Hirose: says Ono was "always working in secrecy".

postdoctoral fellowship at the Carnegie Institution in Washington
DC. Ono, who wanted to be interviewed in Japanese, is shy, serious and reserved.

But their disaffection is not just a clash of personalities. Each researcher has his own version of the discovery of post-perovskite.

Both men agree that advances in technologyled the way. Based on a 1987 report, mostresearchers believed the perovskite form of $\mathrm{MgSiO}_{3}$ was stable down to pressures near the bottom of the mantle, says Hirose". So there seemed little point in looking for post-perovskite. "Not many tried the experiment. It was very 
7 planets. "As theorists we had been waiting for experimental clues," says Artem Oganov, a crystallographer at Swiss Federal Institute of Technology in Zurich. "This stimulated us."

After the first publication by Hirose's group, mineral physicists confirmed, both experimentally and theoretically ${ }^{2-4}$, the existence and structure of post-perovskite $\mathrm{MgSiO}_{3}$. And once researchers started looking at other minerals, such as germanates, which have germanium in place of silicon, they found it there as well. 'It's surprising no one found it earlier, remarks Thomas Duffy, a geophysicist at Princeton University in New Jersey.

Post-perovskite seemed to explain many longstanding mysteries. For instance, simulations by Brodholt and his colleagues tackled the question of why a kind of seismic wave, called shear waves,

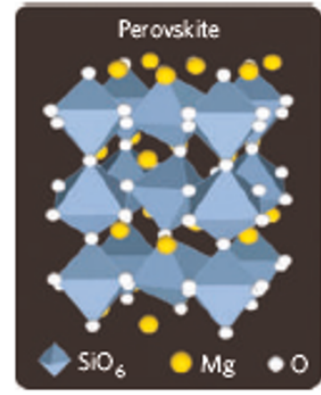

perovskite permits these waves to travel more quickly along certain crystallographic axes than others - giving rise to a pattern of anisotropy that can account for seismic observations in the $\mathrm{D}^{\prime \prime}$ region ${ }^{67}$.

\section{Deep questions}

Such atomic-level studies illuminate bigger questions in Earth science - such as how heat and rock flow from deep in the Earth towards the surface, says Hirose. Figuring out how the lattice structure is deformed as perovskite becomes post-perovskite can tell scientists the

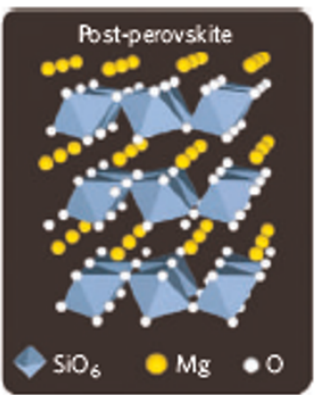

Structures of perovskite (left) and post-perovskite. rate and direction of flow within the lower mantle.

Post-perovskite could help power the great engine of mantle convection in other ways. In some relatively cool regions of the mantle, the phase transition occurs at lower pressure, meaning at a shal-

suddenly accelerates at certain places within the $\mathrm{D}^{\prime \prime}$ region. Shear waves also behave differently there than do compressional waves, another type of seismic wave. If the region contains post-perovskite, rather than perovskite, post-perovskite's properties would explain both observations ${ }^{5}$.

Similarly, post-perovskite can also explain why shear waves travel at different speeds within the $\mathrm{D}^{\prime \prime}$ region depending on their direction, a phenomenon known as seismic anisotropy. The atomic orientation of post-

lower depth. Compared with adjacent warm regions, the newborn post-perovskite is not only cooler but also more dense - giving it another reason to sink ${ }^{\alpha}$ The phase transition is an additional pump helping to drive convection," says Thorne Lay, a seismologist at the University of California, Santa Cruz.

If post-perovskite is really so significant in inner-Earth dynamics, it would be a major coup for the newly discovered structure. But for now, post-perovskite often seems to provide more questions than answers. difficult," says Hirose.

But by 2002 , Hirose could carry out an experiment that he had envisaged more than five years earlier: subjecting a sample of perovskite $\mathrm{MgSiO}_{3}$ to pressures and temperatures near those of the core-mantle boundary. $\mathrm{He}$ analysed the sample at the SPring- 8 synchrotron facility in Hyogo, Japan, and found an odd diffraction pattern in the data.

Formonths, he tried to find a chemist to help out. "Chemistry professors told me there must be something wrong with my data or something wrong with me," says Hirose. Finally Motohiko Murakami, a chemist colleague at Tokyo

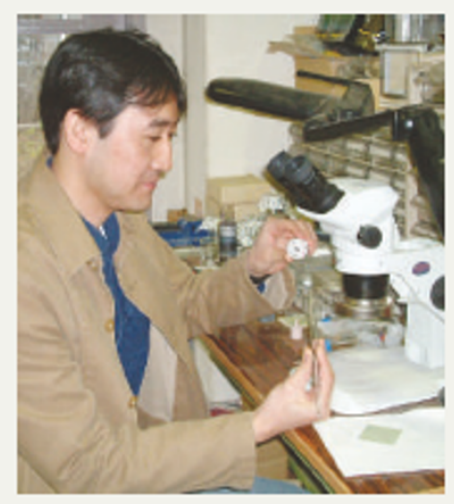

a look. Murakami identified postperovskite in the sample. But Ono says that a key step towards that discovery came more than six months earlier, when he found that the mineral haematite had a high-pressure phase transition - which yielded the same structure that would be found in $\mathrm{MgSiO}_{3}$ by Hirose - and labelled it post-perovskite ${ }^{\mathrm{D}}$. "My objective was always $\mathrm{MgSiO}_{3}{ }^{\prime \prime}$ he says.

Ono says Hirose was ableto follow his work with haematite because researchers using SPring8 are required to post data on a communal computer and an official beam-line notebook. "It wasn't really wrong, but it was
Institute of Technology, found time over his New Year's holiday to take unfair, so I stopped collaborating with him," says Ono. Hirose says Ono "was always working in secrecy". The researchers' colleagues refuse to commenton the row.

The falloutmay have been the inevitable consequence of a number of people converging on a discovery waiting to be made. By 1999 , theorists had already formulated a phase transition with properties that would explain oddities in the $D^{n}$ region of the inner earth. "It was remarkably accurate," says John Brodholt of University College London. "In other words, all it needed was experimental

D.C. evidence."

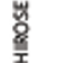

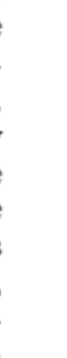




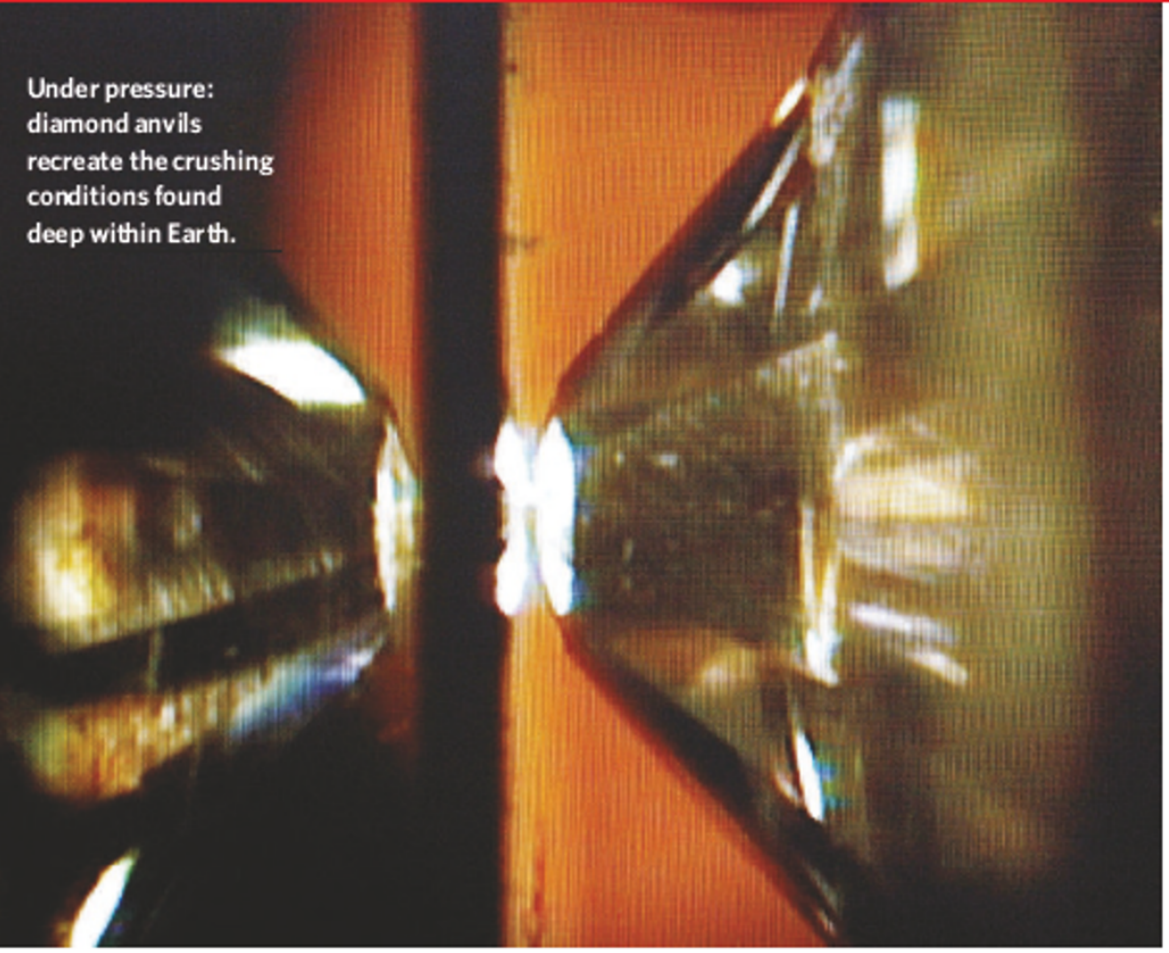

For example, the studies of post-perovskite's role in seismic anisotropy all give different predictions of how the deformed structure would look. Researchers know they must fit the correct deformation pattern into their models - but they don't know which one is right.

Also missing is a precise understanding of the relationship between the pressure and temperature at which the phase transition occurs. The change is known to happen over a specific range of pressure and temperature, with higher temperatures requiring higher pressures. But pressure readings can vary by as much as 15 gigapascals depending on the laboratory equipment used in the experiments translating to a difference in depth of 350 kilometres. ${ }^{\alpha}$ The pressure scale is the largest source of uncertainty", said Hirose last October, at a workshop on post-perovskite in Tokyo.

\section{Spin doctors}

Given that temperature varies greatly over the $D^{\prime \prime}$ region, it is hard to know where postperovskite might show up, argues Paul Tackley, a modeller at the Swiss Federal Institute of Technology in Zurich. His simulations ${ }^{s}$ suggest that a post-perovskite phase would be absent in relatively hot regions, which could be some $3,200^{\circ} \mathrm{C}$, or $1,000^{\circ} \mathrm{C}$ hotter than other regions of the mantle at a similar depth. These are the regions thought to produce mantle plumes, the massive conduits of heat and rock that many think rise from deep in Earth to emerge as 'hotspot' volcanoes on the surface.

Large uncertainties also remain over one of the key questions in Earth dynamics - the extent to which Earth's core of liquid iron interacts with its mantle. Iron atoms can replace some of the magnesium in $\mathrm{MgSiO}_{3}$; Wendy Mao, of the University of Chicago, Illi- nois, has measured just how much iron postperovskite can take up.

The answer, she found, is much more than any previously known lower-mantle material?. "Iron may have a dominant and very complicated role in post-perovskite," she says. Iron absorption may help account for the dramatic slowdown of seismic waves in certain pockets at the base of the mantle. But iron would also broaden the pressure range over which perovskite could turn into postperovskite, making it harder to pinpoint the boundary where the phase transition occurs.

Such problems may arise from trying to understand a complex region with simple models of how post-perovskite behaves, says Lay. ${ }^{\alpha}$ The big issue is that the characterization of post-perovskite is all based on numerical models, he says. And for regions where other elements are intermixed in complex ways, "it is not clear that the numerical models are really relevant to Earth."

The details of the $\mathrm{D}^{\prime \prime}$ zone promise to get knottier before they get simpler. "After the initial euphoria that post-perovskite may explain everything, people are now taking a hard look at its properties," says Mao.

Despite the uncertainties, researchers are pushing into new areas. Shigeaki Ono, of the Japan Marine Science and Technology Center in Yokosuka is investigating $\mathrm{MgSiO}_{3}$ as a possible explanation for one of Earth's mysteries. "Earth's rotation speeds up and slows down a few milliseconds over decade-long periods," says Ono. An electromagnetic coupling between the core and mantle might cause this, but if so the lower mantle must be highly electrically conductive. Perovskite does not have high conductivity, so if the lower mantle is made mostly of perovskite - as many scientists believed - that theory cannot be right.

But Ono has found that a post-perovskite form of $\mathrm{Al}_{2} \mathrm{O}_{3}$ - an aluminium oxide that is better characterized than post-perovskite $\mathrm{MgSiO}_{3}$ - is highly conductive. He argues that post-perovskite $\mathrm{MgSiO}_{3}$ probably has many of the same properties - including a conductivity high enough to explain Earth's rotation discrepancy. "It's a new contribution of the post-perovskite," he says.

\section{Other worlds}

Some researchers have even looked at how post-perovskite $\mathrm{MgSiO}_{3}$ might affect other planets. Renata Wentzcovitch, of the University of Minnesota, Minneapolis, and her colleagues have simulated how post-perovskite would behave inside gas giants or in rocky planets in other solar systems. In such high-pressure, high-temperature fields, they find, the $\mathrm{MgSiO}_{3}$ would start to behave almost like a metal ${ }^{10}$.

Many scientists are excited by the possibility that on Earth there may be perovskite in some regions beneath the post-perovskite zone. This would explain seismic data that suggest shear waves change speed twice, first accelerating and then slowing. It could also constrain the temperature variations to a range of depths. ${ }^{\alpha} \mathrm{It}$ would provide a kind of thermometer of the lower mantle and core," says Thomas.

For his part, Hirose is glad to have provided this useful, if confusing, discovery. Post-perovskite may not account for a large portion of the $\mathrm{D}^{\prime \prime}$ layer, but its existence is what matters. ${ }^{\alpha}$ The proportions might not be huge, he says, "but with its location at the thermal and chemical boundary between the core and the mantle, it is going to help us understand crucial things about the Earth."

\section{David Cyranoski is Nature's Asia-Pacific} correspondent.

1. Murakami, M.etal. Science 304, 855-858 (2004)

2. Oganox A. R. \& Ono, S. Nature 430, 445-448 (2004)

3 Tsuchiya, T.et al. Earth Planet. Sci. Lett. 224, 241-248 (2004).

4. Shim S-Het al. Geophys. Res Lett. 31,L10603(2004).

5. Wookey, J.et al. Nature 438, 1004-1007(2005).

6. Oganok A. Retal. Nature 438, 1142-1144 (2005).

7. Merkel, S.et al. Sdience 311,644-646 (2006).

8. Hernlund I.W. et al. Nature 434, 882-886 (2005).

9. Mao, W.L.etal. Poc Natl Acod. Sci. USA 101, 15867-15869 (2004).

10. Umemota, K etal. Science 311, 983-986 (2006)

11. Knittle, E etal. Science 235, 668 (1987).

12. Ono, S.et a. J. Phys. Chem. Salids 65, 1527-1530 (2004). 